\title{
Clinical Factors Associated with the Risk of Intracranial Aneurysm Rupture in Autosomal Dominant Polycystic Kidney Disease
}

\author{
Chan-Hyuk Lee ${ }^{a, b}$ Curie Ahnc Hyunjin Ryu ${ }^{c}$ Hyun-Seung Kang ${ }^{d}$ \\ Seul-Ki Jeong ${ }^{e}$ Keun-Hwa Jung ${ }^{f}$
}

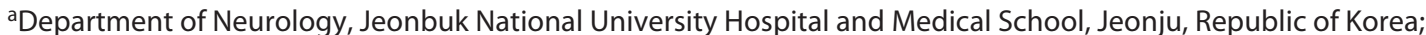
${ }^{b}$ Research Institute of Clinical Medicine of Jeonbuk National University-Biomedical Research Institute of Jeonbuk National University Hospital, Jeonju, Republic of Korea; 'Department of Nephrology, Seoul National University Hospital, Seoul, Republic of Korea; dDepartment of Neurosurgery, Seoul National University Hospital, Seoul, Republic of Korea; 'Visual Intelligence Laboratory, Seul-Ki Jeong Neurology Clinic, Seoul, Republic of Korea; fDepartment of Neurology, Seoul National University Hospital, Seoul National University College of Medicine, Seoul, Republic of Korea
\end{abstract}

\section{Keywords}

Aneurysmal rupture $\cdot$ Autosomal dominant polycystic kidney disease · Dolichoectasia $\cdot$ Hypercholesterolemia . Intracranial aneurysm · Mitral inflow deceleration time

\begin{abstract}
Background: The occurrence of intracranial aneurysms is higher in patients with autosomal dominant polycystic kidney disease (ADPKD) than in the healthy population. However, research concerning the factors related to the risk of intracranial aneurysm rupture in patients with ADPKD is still insufficient. Objectives: The aim of the study was to investigate the prevalence of intracranial aneurysms and aneurysmal subarachnoid hemorrhage (SAH) and to analyze the systemic factors associated with high-risk aneurysms in patients with ADPKD. Methods: We screened patients who underwent cerebral angiography between January 2007 and May 2017 in the ADPKD registry. Patients were examined for the presence of intracranial aneurysms and subsequently reclassified into 3 groups based on the risk of aneurysmal rupture: the aneurysm-negative (group 1), low-risk aneurysm (group 2 ), or high-risk aneurysm (group 3). Various systemic factors
\end{abstract}

karger@karger.com www.karger.com/ced

Karger $\frac{1}{\%}$

GOPEN ACCESS
(C) 2021 The Author(s)

Published by S. Karger AG, Basel

This is an Open Access article licensed under the Creative Commons Attribution-NonCommercial-4.0 International License (CC BY-NC) (http://www.karger.com/Services/OpenAccessLicense), applicable to the online version of the article only. Usage and distribution for commercial purposes requires written permission. were compared, and independent factors associated with high-risk aneurysms were analyzed. Results: Among the 926 patients, $148(16.0 \%)$ had intracranial aneurysms and 11 (1.2\%) had previous aneurysmal SAH. Patients with intracranial aneurysms were further classified into group 2 (low-risk aneurysms, $15.5 \%$ ) or group 3 (high-risk aneurysms, $84.5 \%$ ). Age (odds ratio [OR] 1.03, 95\% confidence interval [CI] 1.01$1.05, p=0.004$ ), female sex (OR 3.13, 95\% Cl 1.94-5.0 6, $p<$ 0.001 ), dolichoectasia (OR 8.57, 95\% Cl 1.53-48.17, $p=0.015$ ), and mitral inflow deceleration time (DT) (OR 1.01, 95\% Cl $1.00-1.01, p=0.046)$ were independently associated with high-risk aneurysms, whereas hypercholesterolemia (OR 0.46 , 95\% Cl 0.29-0.72, $p=0.001$ ) was negatively associated. Conclusion: In the present study among patients with ADPKD, the prevalence of intracranial aneurysms and aneurysmal SAH was 16 and $1.2 \%$, respectively. Age, female sex, dolichoectasia, and mitral inflow DT were positively associated with high-risk aneurysms, whereas hypercholesterolemia was negatively associated. A subsequent large-scaled longitudinal study is needed to define the plausibility of the clinical parameters.

(C) 2021 The Author(s)

Published by S. Karger AG, Basel 


\section{Introduction}

Autosomal dominant polycystic kidney disease (ADPKD) is a genetic disorder, in which multiple cysts invade the renal parenchyma [1]. Mutations of the PKD1 gene on chromosome 16 and $\mathrm{PKD} 2$ gene on chromosome 4 are known to cause ADPKD; PKD1 abnormalities are more common than PKD2 abnormalities, exhibiting nearly $100 \%$ penetrance [2]. Both PKD1 and PKD2 genes are essential in maintaining the integrity of the vascular wall to endure endothelial shear stress. ADPKD has the highest prevalence among single autosomal genetic diseases with 1 in 1,000 of the global population having been diagnosed with ADPKD.

The size and number of renal cysts gradually increase as the disease progresses, with a corresponding increase in the frequency of kidney-related comorbidities such as renal dysfunction, hematuria, and renal hypertension. It generally progresses to ESRD at a median age of 60 years. In addition to the renal complications, patients exhibit several extrarenal complications, including multiple liver and pancreatic cysts, bile duct dilatation, and cardiac valve and vascular insufficiency [3].

Vascular insufficiency includes aneurysms, dissections, and dilatation. Although the prevalence of intracranial aneurysms in patients with ADPKD varies, it is approximately 3-4 times more than in a healthy population, [4] while the incidence of aneurysmal rupture in these patients is $>5$ times that of healthy controls [5]. Aneurysmal rupture accounts for $85 \%$ of subarachnoid hemorrhage (SAH), and half of the patients with untreated $\mathrm{SAH}$ die within a month. Due to the high incidence and probability of aneurysmal rupture in ADPKD, early diagnosis prior to rupture is an essential aspect of patient care [6].

Previous studies have rarely attempted to identify the factors related to the presence of aneurysms and aneurysmal SAH in patients with ADPKD [7]. Herein, we aimed to identify ADPKD-related systemic markers associated with the development and high-risk phenotype of intracranial aneurysms.

\section{Methods}

\section{Study Population}

All the patients who visited a single tertiary hospital were diagnosed with ADPKD between January 1, 2007, and May 31, 2017, and were enrolled in the present study. The diagnostic criteria, as reported by Pei et al. [8], were as follows: patients aged between 15 and 39 years with 3 or more cysts found on 1 or both sides of the renal parenchyma, patients aged between 40 and 59 years with 2 or more cysts found in each kidney, and patients aged 60 years or older with 4 or more cysts in each kidney. The study protocol was approved by the institutional review board of Seoul National University Hospital (No. 1804-054-936). Additionally, the committee waived the requirement for informed consent.

\section{Data Acquisition}

The ADPKD registry primarily aims to assess the target organ damage related to ADPKD. Starting in 2007, we screened newly enrolled patients for demographic and cardiovascular risk factors, and collected their laboratory and echocardiographic data. Patients were classified as hypertensive if they had been diagnosed with hypertension prior to visiting the hospital, were taking antihypertensive drugs, or had at least 2 consecutive blood pressure readings of 140/90 mm Hg. Patients were classified as having diabetes mellitus when they presented with a fasting blood glucose reading of $\geq 126$ $\mathrm{mg} / \mathrm{dL}$, glycated hemoglobin reading of $\geq 6.5$, and random glucose reading of $\geq 200 \mathrm{mg} / \mathrm{dL}$ together with symptoms of hypoglycemia; or when their blood glucose was $\geq 200 \mathrm{mg} / \mathrm{dL}$ following a 75 -g oral glucose tolerance test. Patients were classified as having hypercholesterolemia if they had been diagnosed with hypercholesterolemia prior to their visit to our hospital, or if they were diagnosed after fasting for longer than $8 \mathrm{~h}$ post admission. The diagnostic criteria for hypercholesterolemia during hospitalization were as follows: (a) low-density lipoprotein cholesterol (LDL-C) $\geq 100 \mathrm{mg} / \mathrm{dL}$ with coronary artery disease (CAD) or a CAD equivalent, (b) LDL-C $\geq 130 \mathrm{mg} / \mathrm{dL}$ with 2 or more risk factors, and (c) LDL-C $\geq 160 \mathrm{mg} /$ dL with 1 risk factor-risk factors included smoking, hypertension, high-density lipoprotein cholesterol $<40 \mathrm{mg} / \mathrm{dL}$, and a family history of premature CAD. Patients were classified as having atrial fibrillation if they had been diagnosed prior to admission or when it was observed by means of electrocardiogram or 24-h Holter monitoring during hospitalization. Coronary heart disease was diagnosed when patients had a history of acute myocardial infarction, evidence of asymptomatic myocardial infarction, or myocardial ischemia on an electro- or echocardiogram, or a history of angina pectoris or coronary artery bypass graft. A patient was classified as having a family history of ADPKD if their first-degree relatives had previously been diagnosed with ADPKD.

\section{Subgroup Classification}

First, the patients were classified into 2 groups based on the presence or absence of intracranial aneurysm. Intracranial aneurysms often have a heterogeneous etiology, and certain types of aneurysms (e.g., paraclinoid aneurysm) have a very low risk of rupture [9]. Based on the above, the patients were further classified into 1 of 3 groups: the aneurysm-negative (group 1), low-risk aneurysm (group 2 ), or high-risk aneurysm (group 3). Patients with intracranial aneurysms were classified into group 3, provided they met at least 1 of the following 4 criteria, which were developed and first used by the authors: (1) one or more intracranial aneurysms located distally to the paraophthalmic segment of the internal carotid artery, (2) one or more intracranial aneurysms with a minimum diameter of $5 \mathrm{~mm}$ or more, (3) two or more intracranial aneurysms identified in the same patient, or (4) a history of aneurysmal SAH. Patients who did not meet any of the above criteria were categorized into group 2 .

\section{Classification of Imaging Data}

Dolichoectasia of the basilar artery (BA) was determined based on a cutoff diameter of $4.5 \mathrm{~mm}$ [10]. Blood vessels other than the 
BA were evaluated based on the published diameter cutoff [11]. To minimize the measurement error, each blood vessel was independently measured by 2 researchers, in duplicate, and the average value was used for further analysis. A web-based application was used to measure the total kidney volume [12] and hepatic cysts were graded using previously established criteria [13].

Transthoracic Echocardiography

We evaluated the intracardiac function of patients with ADPKD using several transthoracic echocardiographic measurements. Aortic insufficiency was classified by the presence of an aortic aneurysm and/or dilated ascending aorta; and valvular heart disease as any dysfunction of 1 or more heart valves, including valvular regurgitation or stenosis. In addition, the following values were examined to assess left ventricular (LV) function [14]: early diastolic mitral inflow velocity (mitral inflow E); late diastolic mitral inflow velocity (mitral inflow A); early diastolic mitral annulus velocity (mitral annulus medial E'); mitral inflow deceleration time (DT) - the time interval from the peak of the E-wave to the reference line - LV mass; and LV mass index (LVMI) [15].

\section{Statistical Analysis}

Demographic, laboratory, and imaging data were compared between the 2 groups using Pearson's $\chi^{2}$ and Student's $t$ test for categorical and continuous variables, respectively. When comparing the 3 groups, one-way ANOVA and the $\chi^{2}$ test for trend were used for continuous and categorical variables, respectively. To identify the factors affecting the risk of aneurysmal rupture, multinomial logistic regression was used to estimate the adjusted odds ratio (OR) of each variable. A two-sided $p$ value of $<0.05$ was considered statistically significant. All statistical analyses were performed using SPSS 25.0 for Windows (IBM Corp., Armonk, NY, USA).

\section{Results}

In total, 1,666 patients diagnosed with ADPKD from January 2007 to May 2017 were screened in this study, from which $740(44.4 \%)$ were excluded because their cerebrovascular images were not available. Thereupon, 926 patients were included in the final analysis. Among them, $148(16.0 \%)$ presented with a total of 195 intracranial aneurysms. Intracranial aneurysms were most frequently observed in the middle cerebral artery (45.6\%), and the proportion of aneurysms with a diameter $\geq 5 \mathrm{~mm}$ was $16.0 \%$, as shown in Table 1 . Approximately, 11 patients $(1.2 \%$ of the total number of patients and $7.4 \%$ of patients with intracranial aneurysms) had a previous history of aneurysmal SAH.

The aneurysm-positive group comprised older patients and a higher proportion of women than the aneurysm-negative group (Table 2). Additionally, the aneurysm-positive group had a higher prevalence of hypertension and intracranial dolichoectasia, higher grade of
Table 1. Location and size of intracranial aneurysms in ADPKD patients

\begin{tabular}{ll}
\hline & $\begin{array}{l}\text { Intracranial aneurysms, } \\
n(\%)\end{array}$ \\
\hline Location* & \\
Anterior communicating artery & $17(8.7)$ \\
Anterior cerebral artery & $10(5.1)$ \\
Middle cerebral artery & $89(45.6)$ \\
Posterior communicating artery & $13(6.7)$ \\
Internal cerebral artery & $47(24.1)$ \\
Posterior arteries & \\
Size in largest dimension, mm & $\oint$ \\
$<2.0$ & $19(9.7)$ \\
$2.0-2.9$ & $14(7.5)$ \\
$3.0-3.9$ & $69(36.9)$ \\
$4.0-4.9$ & $52(27.8)$ \\
$5.0-6.9$ & $22(11.8)$ \\
$7.0-9.9$ & $16(8.6)$ \\
$10.0-12.9$ & $10(5.3)$ \\
$>13.0$ & $3(1.6)$ \\
& $1(0.5)$
\end{tabular}

ADPKD, autosomal dominant polycystic kidney disease; BA, basilar artery; MRA, magnetic resonance angiography. ${ }^{*}$ The total number of IAs is 195 in 148 patients. ${ }^{\dagger}$ Posterior arteries include posterior cerebral artery and BA. ${ }^{\Phi}$ Among the total IAs, 187 aneurysms in 142 patients were measured for the dimension, but 8 aneurysms in 6 patients were not because the aneurysms were obscured in MRA.

hepatic cysts, larger total kidney volume, and a more prolonged mitral inflow DT than the aneurysm-negative group. Hypercholesterolemia was observed less frequently in the aneurysm-positive group than the aneurysmnegative group.

Patients with intracranial aneurysms were further classified into subgroups according to the risk of rupture (as described in methods section): 23 (15.5\%) patients were assigned to the low-risk aneurysm group (group 2), and $125(84.5 \%)$ to the high-risk aneurysm group (group 3 ; Table 3). Group 3 comprised older patients and a higher proportion of women, along with a higher prevalence of dolichoectasia, larger total kidney volume, higher LVMI and mitral inflow DT, and a lower glomerular filtration rate than the aneurysm-negative group. The aneurysm-negative group had a higher proportion of patients with hypercholesterolemia than the other 2 groups. Multinomial logistic regression analysis (Table 4) identified age (OR 1.03, 95\% confidence interval (CI) 1.01$1.05, p=0.004$ ), female sex (OR 3.13, 95\% CI 1.94-5.06, $p<0.001$ ), hypercholesterolemia (OR 0.46, 95\% CI 0.29$0.72, p=0.001$ ), dolichoectasia (OR 8.57, 95\% CI 1.53- 
Table 2. Characteristics according to the presence of intracranial aneurysms in ADPKD patients

\begin{tabular}{|c|c|c|c|}
\hline \multirow[t]{2}{*}{ Variables } & \multicolumn{2}{|c|}{ Intracranial aneurysm } & \multirow[t]{2}{*}{$p$ value } \\
\hline & negative $(N=778)$ & positive $(N=148)$ & \\
\hline \multicolumn{4}{|l|}{ Demographics } \\
\hline Age, years & $50.8 \pm 13.5$ & $57.0 \pm 12.2$ & $<0.001$ \\
\hline Male & $389(50.0)$ & $45(30.4)$ & $<0.001$ \\
\hline \multicolumn{4}{|l|}{ CVD risk factors } \\
\hline Hypertension & $568(73.0)$ & $121(81.8)$ & 0.025 \\
\hline Diabetes mellitus & $51(6.6)$ & $8(5.4)$ & 0.600 \\
\hline Hypercholesterolemia & $305(39.2)$ & $42(28.4)$ & 0.013 \\
\hline Atrial fibrillation & $14(1.8)$ & $2(1.4)$ & 1.000 \\
\hline Smoking & $75(9.6)$ & $9(6.1)$ & 0.167 \\
\hline Alcohol consumption & $91(11.7)$ & $18(12.2)$ & 0.872 \\
\hline Ischemic heart disease & $46(5.9)$ & $3(2.0)$ & 0.068 \\
\hline BMI & $23.1 \pm 3.1$ & $22.9 \pm 2.6$ & 0.522 \\
\hline Family history & $374(48.1)$ & $64(43.2)$ & 0.281 \\
\hline \multicolumn{4}{|l|}{ Echocardiography } \\
\hline Aortic insufficiency & $32(4.1)$ & $7(4.7)$ & 0.732 \\
\hline Valvular heart disease & $150(19.3)$ & $32(21.6)$ & 0.511 \\
\hline Aortic diameter, mm & $33.9 \pm 3.2$ & $33.7 \pm 2.7$ & 0.446 \\
\hline Mitral inflow E, m/s & $0.6 \pm 2.5$ & $0.5 \pm 0.1$ & 0.667 \\
\hline Mitral inflow $\mathrm{A}, \mathrm{m} / \mathrm{s}$ & $0.6 \pm 0.2$ & $0.6 \pm 0.2$ & 0.147 \\
\hline Mitral inflow DT, ms & $198.7 \pm 38.7$ & $210.9 \pm 45.2$ & 0.002 \\
\hline Mitral inflow E/A & $0.9 \pm 0.4$ & $0.9 \pm 0.3$ & 0.043 \\
\hline Mitral annulus medial E', $\mathrm{cm} / \mathrm{s}$ & $6.5 \pm 1.6$ & $6.1 \pm 1.5$ & 0.007 \\
\hline $\mathrm{MV} \mathrm{E} / \mathrm{E}^{\prime}$ & $8.6 \pm 2.4$ & $9.3 \pm 3.1$ & 0.010 \\
\hline LV mass, $\mathrm{g}$ & $150.3 \pm 32.9$ & $154.4 \pm 43.2$ & 0.266 \\
\hline LVMI, g/m² & $86.9 \pm 17.9$ & $91.4 \pm 27.0$ & 0.055 \\
\hline Dolichoectasia & $2(0.3)$ & $5(3.4)$ & $<0.001$ \\
\hline \multicolumn{4}{|l|}{ Disease severity } \\
\hline Total KV, L & $1.8 \pm 1.6$ & $2.3 \pm 1.8$ & 0.003 \\
\hline $\mathrm{KV}$, right, $\mathrm{mL}$ & $0.9 \pm 0.8$ & $1.1 \pm 0.9$ & 0.002 \\
\hline $\mathrm{KV}$, left, mL & $0.9 \pm 0.9$ & $1.2 \pm 1.0$ & 0.008 \\
\hline Liver cysts [IQR] & $3[1-3]$ & $3[3-3]$ & $<0.001$ \\
\hline \multicolumn{4}{|l|}{ Laboratory } \\
\hline $\mathrm{WBC}, \times 10^{3} / \mu \mathrm{L}$ & $6.2 \pm 2.1$ & $5.9 \pm 2.6$ & 0.107 \\
\hline Neutrophil, \% & $58.8 \pm 9.9$ & $60.7 \pm 10.9$ & 0.043 \\
\hline Lymphocyte, \% & $30.7 \pm 9.2$ & $29.3 \pm 10.0$ & 0.089 \\
\hline AST, IU/L & $21.0 \pm 17.2$ & $21.5 \pm 12.8$ & 0.785 \\
\hline $\mathrm{ALT}, \mathrm{U} / \mathrm{L}$ & $18.2 \pm 13.5$ & $16.2 \pm 9.7$ & 0.089 \\
\hline Total bilirubin, mg/dL & $0.8 \pm 1.0$ & $0.8 \pm 1.2$ & 0.915 \\
\hline $\mathrm{Cr}, \mathrm{mg} / \mathrm{dL}$ & $2.2 \pm 4.9$ & $2.4 \pm 2.5$ & 0.564 \\
\hline $\mathrm{GFR}, \mathrm{mL} / \mathrm{min} / 1.73 \mathrm{~m}^{2}$ & $62.2 \pm 33.3$ & $48.2 \pm 32.5$ & $<0.001$ \\
\hline $\mathrm{ESR}, \mathrm{mm} / \mathrm{h}$ & $16.4 \pm 14.6$ & $19.2 \pm 18.9$ & 0.095 \\
\hline
\end{tabular}

ADPKD, autosomal dominant polycystic kidney disease; CVD, cardiovascular disease; DT, deceleration time; LV, left ventricular; LVMI, left ventricular mass index; KV, kidney volume; WBC, white blood cell; AST, aspartate amino transferase; ALT, amino alanine transferase; GFR, glomerular filtration rate; ESR, erythrocyte sedimentation rate. A $p$ value $<0.05$ is statistically significant.

$48.17, p=0.015$ ), and mitral inflow DT (OR 1.01, 95\% CI $1.00-1.01, p=0.046)$ as the factors significantly associated with high-risk aneurysms. In contrast, none of the factors were associated with low-risk aneurysms when compared to the aneurysm-negative group. Logistic re- gression analysis according to aneurysmal rupture (group 1 and 2 vs. group 3) showed similar results to multinomial logistic regression analysis (group 1 vs. group 2 vs. group 3; online suppl. Table; for all online suppl. material, see www.karger.com/doi/10.1159/000513709). 
Table 3. Characteristics according to the presence and risk of intracranial aneurysms in ADPKD patients

\begin{tabular}{|c|c|c|c|c|}
\hline \multirow[t]{2}{*}{ Variables } & \multicolumn{3}{|c|}{ Intracranial aneurysm } & \multirow[t]{2}{*}{$p$ value } \\
\hline & negative $(N=778)$ & low risk $(N=23)$ & high risk $(N=125)$ & \\
\hline \multicolumn{5}{|l|}{ Demographics } \\
\hline Age, years & $50.8 \pm 13.5$ & $52.0 \pm 14.3$ & $57.9 \pm 11.6$ & $<0.001^{*}$ \\
\hline Male, $n(\%)$ & $389(50.0)$ & $15(65.2)$ & $30(24.0)$ & $<0.001^{* *}$ \\
\hline \multicolumn{5}{|l|}{ CVD risk factors } \\
\hline Hypertension & $568(73.0)$ & $17(73.9)$ & $104(83.2)$ & 0.053 \\
\hline Diabetes mellitus & $51(6.6)$ & $1(4.3)$ & $7(5.6)$ & 0.850 \\
\hline Hypercholesterolemia & $305(39.2)$ & $6(26.1)$ & $36(28.8)$ & 0.043 \\
\hline Atrial fibrillation & $14(1.8)$ & $0(0.0)$ & $2(1.6)$ & 0.803 \\
\hline Smoking & $75(9.6)$ & $3(13.0)$ & $6(4.8)$ & 0.173 \\
\hline Alcohol consumption & $91(11.7)$ & $4(17.4)$ & $14(11.2)$ & 0.690 \\
\hline Ischemic heart disease & $46(5.9)$ & $0(0.0)$ & $3(2.4)$ & 0.138 \\
\hline BMI & $23.1 \pm 3.1$ & $23.3 \pm 2.1$ & $22.8 \pm 2.6$ & 0.643 \\
\hline Family history & $374(48.1)$ & $11(47.8)$ & $53(42.4)$ & 0.498 \\
\hline \multicolumn{5}{|l|}{ Echocardiography } \\
\hline Aortic insufficiency & $32(4.1)$ & $1(4.3)$ & $6(4.8)$ & 0.939 \\
\hline Valvular heart disease & $150(19.3)$ & $4(17.4)$ & $28(22.4)$ & 0.691 \\
\hline Aortic diameter, mm & $33.9 \pm 3.2$ & $35.0 \pm 3.0$ & $33.5 \pm 2.6$ & 0.075 \\
\hline Mitral inflow E, m/s & $0.6 \pm 2.5$ & $0.5 \pm 0.2$ & $0.5 \pm 0.1$ & 0.093 \\
\hline Mitral inflow $\mathrm{A}, \mathrm{m} / \mathrm{s}$ & $0.6 \pm 0.2$ & $0.6 \pm 0.1$ & $0.7 \pm 0.2$ & 0.104 \\
\hline Mitral inflow DT, ms & $198.7 \pm 38.7$ & $211.6 \pm 31.6$ & $210.7 \pm 47.3$ & $0.003^{*}$ \\
\hline Mitral inflow E/A & $0.9 \pm 0.4$ & $1.0 \pm 0.3$ & $0.9 \pm 0.3$ & 0.110 \\
\hline Mitral annulus medial E', $\mathrm{cm} / \mathrm{s}$ & $6.5 \pm 1.6$ & $6.9 \pm 1.8$ & $6.0 \pm 1.4$ & $0.001^{* *}$ \\
\hline MV E/E' & $8.6 \pm 2.4$ & $8.2 \pm 2.2$ & $9.5 \pm 3.2$ & $0.001^{* *}$ \\
\hline LV mass, g & $150.3 \pm 32.9$ & $158.8 \pm 25.6$ & $153.6 \pm 45.8$ & 0.328 \\
\hline LVMI, $\mathrm{g} / \mathrm{m}^{2}$ & $86.9 \pm 17.9$ & $89.8 \pm 12.1$ & $91.7 \pm 29.0$ & $0.037^{*}$ \\
\hline Dolichoectasia & $2(0.3)$ & $0(0.0)$ & $5(4.0)$ & $<0.001^{*}$ \\
\hline \multicolumn{5}{|l|}{ Disease severity } \\
\hline Total KV, L & $1.8 \pm 1.6$ & $1.6 \pm 1.2$ & $2.4 \pm 1.9$ & $<0.001^{*}$ \\
\hline Liver cysts & $2.4 \pm 1.2$ & $2.2 \pm 1.3$ & $3.0 \pm 0.9$ & $<0.001^{* *}$ \\
\hline \multicolumn{5}{|l|}{ Laboratory } \\
\hline $\mathrm{WBC}, \times 10^{3} / \mu \mathrm{L}$ & $6.2 \pm 2.1$ & $6.2 \pm 1.5$ & $5.8 \pm 2.7$ & 0.200 \\
\hline Neutrophil, \% & $58.8 \pm 9.9$ & $58.8 \pm 9.6$ & $61.0 \pm 11.2$ & 0.079 \\
\hline Lymphocyte, \% & $30.7 \pm 9.2$ & $30.0 \pm 8.8$ & $29.1 \pm 10.2$ & 0.217 \\
\hline AST, IU/L & $21.0 \pm 17.2$ & $18.4 \pm 4.7$ & $22.0 \pm 13.7$ & 0.616 \\
\hline ALT, IU/L & $18.2 \pm 13.5$ & $14.3 \pm 7.0$ & $16.6 \pm 10.1$ & 0.175 \\
\hline Total bilirubin, $\mathrm{mg} / \mathrm{dL}$ & $0.8 \pm 1.0$ & $0.9 \pm 0.6$ & $0.8 \pm 1.3$ & 0.877 \\
\hline $\mathrm{Cr}, \mathrm{mg} / \mathrm{dL}$ & $2.2 \pm 4.9$ & $1.8 \pm 1.8$ & $2.5 \pm 2.6$ & 0.654 \\
\hline $\mathrm{GFR}, \mathrm{mL} / \mathrm{min} / 1.73 \mathrm{~m}^{2}$ & $62.2 \pm 33.3$ & $58.9 \pm 30.4$ & $46.2 \pm 32.6$ & $<0.001^{*}$ \\
\hline $\mathrm{ESR}, \mathrm{mm} / \mathrm{h}$ & $16.4 \pm 14.6$ & $21.0 \pm 30.0$ & $18.9 \pm 16.2$ & 0.112 \\
\hline
\end{tabular}

ADPKD, autosomal dominant polycystic kidney disease; CVD, cardiovascular disease; DT, deceleration time; LV, left ventricular; LVMI, left ventricular mass index; KV, kidney volume; WBC, white blood cell; AST, aspartate amino transferase; ALT, amino alanine transferase; GFR, glomerular filtration rate; ESR, erythrocyte sedimentation rate. ${ }^{*}$ Significant difference between the negative and high-risk groups. ${ }^{* *}$ Significant difference between the high-risk group and others.

\section{Discussion}

The prevalence of intracranial aneurysms in patients with ADPKD is reported to be approximately $10-12 \%$, [16] ranging from 1.2\% (Finnish) to 20\% (Japanese), which is around 3 times higher than in the general population. In the present study, the prevalence was $16 \%$ in patients with ADPKD, which is 3 times higher than in the general population from the Republic of Korea. 
Table 4. Multinomial regression analysis according to rupture risk of intracranial aneurysms in ADPKD patients

\begin{tabular}{lllr}
\hline Aneurysm & Variables & OR (95\% CI) & \multicolumn{1}{c}{$\begin{array}{l}p \\
\text { value* }\end{array}$} \\
\hline $\begin{array}{lllr}\text { Group 1 } \\
\text { (No aneurysm) }\end{array}$ & Reference & \\
Group 2 & None & & \\
Group 3 & Age, year & $1.03(1.01-1.05)$ & 0.004 \\
& Female & $3.13(1.94-5.06)$ & $<0.001$ \\
& Hypercholesterolemia & $0.46(0.29-0.72)$ & 0.001 \\
& Dolichoectasia & $8.57(1.53-48.17)$ & 0.015 \\
& Mitral inflow DT, ms & $1.01(1.00-1.01)$ & 0.046 \\
\hline
\end{tabular}

Group 2 included the ADPKD patients with intracranial aneurysms of low risk and group 3 included the ADPKD patients with intracranial aneurysms of high risk. OR, odds ratio; CI, confidence interval; GFR, glomerular filtration rate; DT, deceleration time. * Adjusted by age, sex, hypertension, hypercholesterolemia, dolichoectasia, mitral inflow DT, total kidney volume, GFR, and grade for liver cysts.

Approximately, $80 \%$ of spontaneous SAHs are caused by aneurysmal rupture [17]. A meta-analysis of 18 studies showed that the annual incidence of spontaneous SAH in the general population was $0.01 \%$. In contrast, aneurysmal SAH in patients with ADPKD was reported to be 4 times higher than in controls, occurring at a younger age and comprising relatively small-sized aneurysms. Among patients undergoing kidney transplantation, patients with ADPKD had a prevalence of SAH that was 4 times higher than patients without ADPKD [6]. In the present study, the prevalence of previous aneurysmal SAH in patients with ADPKD was $1.2 \%$, which is notably higher than in the general population $(0.02 \%)$ of Korea. However, there was no difference in the outcome of aneurysmal SAH between the patients with ADPKD and age- and sex-matched controls [18].

Intracranial arterial dolichoectasia occurs when the diameter of at least 1 major cerebrovascular artery is extended beyond its normal range. A previous study showed that intracranial dolichoectasia was present in $4.7 \%$ of patients with ADPKD, approximately 80 times higher than in the control group $(0.06 \%)$. In another study of patients with ADPKD who underwent cerebral angiography, dolichoectasia was reported in $2.3 \%$ of patients, but not in patients without ADPKD [19]. In the present study, 7 patients $(0.76 \%)$ presented with intracranial dolichoectasia, which is lower than in previous studies. Moreover, intracranial dolichoectasia was mainly found in patients with aneurysm (3.4\%), particularly those with a high-risk phenotype (4.0\%). Vascular abnormalities of intracranial an- eurysms and dolichoectasia originate from the loss of wall integrity as a result of weakened connective tissue. The manifesting features of abnormal vascular wall integrity differ depending on the vascular geometry: dolichoectasia occurs in a long cylindrical artery such as the BA, and aneurysms occur at sites of bifurcation or branching. However, whether the distribution of intracranial dolichoectasia or aneurysms differs according to vascular geometry or ethnicity requires further investigation.

Patients with ADPKD have a higher incidence of heart disease than healthy individuals, which is one of the leading causes of death in these patients [20]. One in 4 patients with ADPKD present with mitral valve prolapse, and aortic root dilatation has been reported in $8 \%$. Additionally, patients with ADPKD have a high incidence rate of LV hypertrophy. Mitral inflow DT refers to the time interval between the early diastolic stage of the left ventricle and the final deceleration [21]. In grade $1 \mathrm{LV}$ diastolic dysfunction, initial filling of the ventricle is limited. Therefore, mitral inflow DT tends to extend, while in grade 2 or higher, the pressure in the left atrium becomes greater than in the left ventricle, and the mitral inflow DT decreases more than normal. The variable pattern of mitral inflow DT partly reflects the LV diastolic dysfunction. Further research is warranted to determine whether patients with aneurysms and a long mitral inflow DT progress to severe LV dysfunction [22].

In the present study, patients with hypercholesterolemia had a lower prevalence of intracranial and high-risk aneurysms. Low serum cholesterol or LDL-C is associated with increased hemorrhagic stroke [23]. In a metaanalysis of intracranial aneurysms, a high serum cholesterol was associated with a low risk of SAH. Meanwhile in other studies, a high total cholesterol concentration increased the risk of intracerebral hemorrhage and $\mathrm{SAH}$ [24]. Large-scale prospective studies are needed to establish whether hypercholesterolemia has a protective effect on intracranial aneurysms and aneurysmal SAH in patients with ADPKD.

The categorization of the patients with ADPKD according to the 4 criteria seems to be arbitrary in the present study. However, it deserves mentioning that the lowrisk aneurysm group (group 2) showed numerically intermediate (such as LVMI and GFR) or relatively healthy characteristics (such as dolichoectasia, kidney volume, and hepatic cysts). Although further studies are needed, a simple set of 4 criteria (location, maximal width, multiplicity, and a history of SAH) may be helpful to screen for high-risk intracranial aneurysms in patients with ADPKD. 
This study has a few limitations. First, it is a retrospective cross-sectional study. Over $40 \%$ of the initially screened patients were excluded due to the unavailability of their cerebrovascular images. A longitudinal prospective study is needed in the future to define whether intracranial aneurysms evolve into high-risk aneurysms or aneurysmal SAH. Second, the present study was performed in a single center; therefore, the generalizability of the findings cannot be guaranteed. A multicenter study is needed to enhance the applicability of the findings to a larger population. Last, magnetic resonance angiography (MRA) was used to detect aneurysms and dolichoectasia. MRA is known to underestimate the arterial diameter, and this may have affected the reported prevalence of dolichoectasia.

\section{Conclusion}

In the present study among patients with ADPKD, the prevalence of intracranial aneurysms and aneurysmal SAH was 16 and 1.2\%, respectively. Age, female sex, dolichoectasia, and mitral inflow DT were significantly and positively associated with high-risk aneurysms, whereas hypercholesterolemia was negatively associated. Metabolic parameters have to be carefully examined and considered for the suitable care of patients with ADPKD.

\section{Statement of Ethics}

The study has been approved by the research institute's committee on human research, and the committee waived the requirement for informed consent. (Seoul National University Hospital; No. 1804-054-936).

\section{Conflict of Interest Statement}

The authors declare that there is no conflict of interest.

\section{Funding Sources}

This work was supported by the National Research Foundation of Korea (NRF) grant funded by the Korea government (MSIT) (2020R1A2C1100337). Jung K.H. was supported by a research grant from Chong Kun Dang, Pharm (0620161560).

\section{Author Contributions}

K.H.J. and C.H.L. contributed to study design and conception. S.K.J. and C.H.L. performed the statistical analysis and interpreted the data. C.H.L. wrote the first draft of the manuscript. C.A. and H.J.R. revised the manuscript for intellectual content. H.S.K. provided critical advice and comments for the manuscript. All authors read and approved the final manuscript.

\section{References}

1 Iglesias CG, Torres VE, Offord KP, Holley KE, Beard CM, Kurland LT. Epidemiology of adult polycystic kidney disease, Olmsted county, Minnesota: 1935-1980. Am J Kidney Dis. 1983;2(6):630-9.

2 Srivastava A, Patel N. Autosomal dominant polycystic kidney disease. Am Fam Physician. 2014;90(5):303-7.

3 Luciano RL, Dahl NK. Extra-renal manifestations of autosomal dominant polycystic kidney disease (ADPKD): considerations for routine screening and management. Nephrol Dial Transplant. 2014;29(2):24754.

4 Xu HW, Yu SQ, Mei CL, Li MH. Screening for intracranial aneurysm in 355 patients with autosomal-dominant polycystic kidney disease. Stroke. 2011;42(1):204-6.

5 Schievink WI, Torres VE, Piepgras DG, Wiebers DO. Saccular intracranial aneurysms in autosomal dominant polycystic kidney disease. J Am Soc Nephrol. 1992;3(1): 88-95.

6 Cheungpasitporn W, Thongprayoon C, Ungprasert P, Wijarnpreecha K, Kaewput W, Lee-

Factors Affecting High-Risk Aneurysm in ADPKD aphorn N, et al. Subarachnoid hemorrhage in hospitalized renal transplant recipients with autosomal dominant polycystic kidney disease: a nationwide analysis. J Clin Med. 2019; 8(4):524.

7 Sanchis IM, Shukoor S, Irazabal MV, Madsen CD, Chebib FT, Hogan MC, et al. Presymptomatic screening for intracranial aneurysms in patients with autosomal dominant polycystic kidney disease. Clin J Am Soc Nephrol. 2019;14(8):1151-60

8 Pei Y, Obaji J, Dupuis A, Paterson AD, Magistroni R, Dicks E, et al. Unified criteria for ultrasonographic diagnosis of ADPKD. J Am Soc Nephrol. 2009;20(1):205-12.

9 Jeon JS, Ahn JH, Huh W, Son YJ, Bang JS, Kang HS, et al. A retrospective analysis on the natural history of incidental small paraclinoid unruptured aneurysm. J Neurol Neurosurg Psychiatry. 2014;85(3):289-94.

10 Smoker WR, Price MJ, Keyes WD, Corbett JJ, Gentry LR. High-resolution computed tomography of the basilar artery: 1 . Normal size and position. AJNR Am J Neuroradiol. 1986; 7(1):55-60.
11 Passero SG, Rossi S. Natural history of vertebrobasilar dolichoectasia. Neurology. 2008; 70(1):66-72.

12 Research MFaMEa. Imaging classification of ADPKD: a simple model for selecting patients for clinical trials. Scottsdale: Mayo Foundation and Medical Education and Research; 2013.

13 Qian Q, Li A, King BF, Kamath PS, Lager DJ, Huston J 3rd, et al. Clinical profile of autosomal dominant polycystic liver disease. Hepatology. 2003;37(1):164-71.

14 Nagueh SF, Smiseth OA, Appleton CP, Byrd BF 3rd, Dokainish H, Edvardsen T, et al. Recommendations for the evaluation of left ventricular diastolic function by echocardiography: an update from the American society of echocardiography and the European association of cardiovascular imaging. J Am Soc Echocardiogr. 2016;29(4):277-314.

15 Liao MT, Liao CW, Tsai CH, Chang YY, Chen ZW, Pan CT, et al. U-shaped relationship between left ventricular mass index and estimated glomerular filtration rate in patients with primary aldosteronism. J Investig Med. 2020; 68(2):371-7. 
16 Zhou Z, Xu Y, Delcourt C, Shan J, Li Q, Xu J, et al. Is regular screening for intracranial aneurysm necessary in patients with autosomal dominant polycystic kidney disease? A systematic review and meta-analysis. Cerebrovasc Dis. 2017;44(1-2):75-82.

17 Kassell NF, Torner JC, Jane JA, Haley EC Jr, Adams HP. The international cooperative study on the timing of aneurysm surgery. Part 2: surgical results. J Neurosurg. 1990;73(1):37-47.

18 Nurmonen HJ, Huttunen T, Huttunen J, Kurtelius A, Kotikoski S, Junkkari A, et al. Lack of impact of polycystic kidney disease on the outcome of aneurysmal subarachnoid hemorrhage: a matched case-control study. J Neurosurg. $2020 \mathrm{Jul}$ 3:1-8.
19 Schievink WI, Torres VE, Wiebers DO, Huston J 3rd. Intracranial arterial dolichoectasia in autosomal dominant polycystic kidney disease. J Am Soc Nephrol. 1997;8(8):1298303.

20 Perrone RD, Ruthazer R, Terrin NC. Survival after end-stage renal disease in autosomal dominant polycystic kidney disease: contribution of extrarenal complications to mortality. Am J Kidney Dis. 2001;38(4):777-84.

21 Vermeiren GL, Malbrain ML, Walpot JM. Cardiac ultrasonography in the critical care setting: a practical approach to asses cardiac function and preload for the "non-cardiologist." Anaesthesiol Intensive Ther. 2015;47 Spec No:s89-104.
22 Spinelli L, Pisani A, Giugliano G, Trimarco B, Riccio E, Visciano B, et al. Left ventricular dysfunction in ADPKD and effects of octreotide-LAR: a cross-sectional and longitudinal substudy of the ALADIN trial. Int J Cardiol. 2019;275:145-51.

23 Zhang Y, Tuomilehto J, Jousilahti P, Wang Y, Antikainen R, Hu G. Total and high-density lipoprotein cholesterol and stroke risk. Stroke. 2012;43(7):1768-74.

24 Lindbohm JV, Kaprio J, Korja M. Cholesterol as a risk factor for subarachnoid hemorrhage: a systematic review. PLoS One. 2016;11(4): e0152568. 